\title{
La modulación de Políticas Públicas en el Estado contemporáneo: condicionamientos, incompatibilidades y racionalidad sistémica
}

\author{
DIMAS SANTIBÁÑEZ YÁÑEZ \\ Antropólogo Social, Universidad de Chile \\ dsantiba@uchile.cl
}

\section{Presentación}

Una especie de incomodidad recorre de modo persistente los balances y evaluaciones que se realizan sobre los éxitos y fracasos del modelo de modernización que Chile ha privilegiado. Parte de esta incomodidad, se aprecia en los esfuerzos que todavía se realizan para reafirmar la discontinuidad que se ha buscado producir en la experiencia chilena a partir de las políticas implementadas por los gobiernos democráticos ${ }^{[1]}$. Después de tanta precisión, no queda más que concluir que ya son algo más de treinta años de continuidad en la orientación del modelo de desarrollo económico y social.

Por cierto, no todo es igual. Las estrategias de crecimiento y las recetas macroeconómicas, han debido sufrir correcciones y ajustes y se han incrementado los esfuerzos orientados a generar mayores y mejores condiciones de equidad social. Sin embargo, en lo sustantivo no se han modificado las premisas que sostienen y condicionan las decisiones que las distintas administraciones han debido adoptar a lo largo del tiempo. El abanico de políticas que dan vida a las estrategias de desarrollo, responde a un conjunto de concepciones y fórmulas más o menos acotadas que imponen limitaciones a su diseño, a su selección y priorización. Desde mi perspectiva, este tipo de condicionamientos buscan ser orientados desde criterios particularizados de racionalidad sistémica, lo cual suele ser indicado como una medida del déficit de racionalidad global. Solo en ese tipo de contextos,

[1] Martner, Gonzalo. Remodelar el modelo. Reflexiones para el Bicentenario. LOM Ediciones, Santiago 2007. 
es posible la comunicación que busca discriminar entre matices ideológicos o disparidades en las consideraciones técnicas.

La incomodidad o el malestar, no es algo nuevo en la descripción de las transformaciones sociales y culturales de nuestro país y, en algún sentido, han hecho referencia al déficit de racionalidad de la sociedad ${ }^{[2]}$. Las ciencias sociales y la política, han coincido en más de alguna ocasión en torno a este diagnóstico. Progresivamente, en la forma inclusión/exclusión se ha venido constituyendo un espacio de comunicación lo suficientemente abstracto y motivador como para que la sociedad, la economía y la política, así como las ciencias sociales, encuentren un margen para la comprensión y aceptación de indicaciones y, en consecuencia, para una eventual co-orientación. La abstracción de la forma inclusión/exclusión conduce al observador a la paradoja de la relevancia/irrelevancia de las personas, en el marco de la diferenciación de la sociedad ${ }^{[3]}$. Dicho en otros términos, de la manera en que las personas se vuelven observables debido a las condiciones de opacidad o marginalidad en que las sitúan las transformaciones socio-estructurales y, con ello, a la aceptación de la incapacidad de la sociedad por regular una inclusión uniforme y universal al conjunto de prestaciones que generan los sistemas de funciones ${ }^{[4]}$. Por ello, y a pesar de las ventajas teóricas de la forma, no se puede ni se debe olvidar que el tratamiento de las condiciones de exclusión exige precisiones específicas: desigualdad económica, inequidad en la educación, deficiencias en el acceso a la salud, baja calidad de la vivienda, por nombrar las más relevantes. En el caso de Chile, la comunicación de la sociedad traslada progresivamente el malestar desde la cuantificación de las

[2] Al menos desde el Informe Desarrollo Humano del Programa de las Naciones Unidas para el Desarrollo. Las Paradojas de la Modernización. Santiago de Chile, 1998.

[3] Sobre el problema teórico, Luhmann, Niklas. Complejidad y modernidad. De la unidad a la diferencia. Editorial Trotta, Madrid, 1998: 167 y ss. También, Robles, Fernando. "La no-observación de la inclusión-exclusión. Autopoiesis, tiempo y exclusión social”. En: Nuevas Exclusiones en la complejidad social contemporánea. Ediciones MAD, Facultad de Ciencias Sociales Universidad de Chile, 2006.

[4] Sobre la visibilidad/ invisibilidad, y el carácter multidimensional y dinámico de la exclusión social en América Latina: Banco Interamericano de Desarrollo. ¿Los de afuera? Patrones cambiantes de exclusión en América Latina y el Caribe. Informe 2008. IPES, BID, Harvard University. Washington: Estados Unidos, 2007. 
personas en condiciones de pobreza, a la brecha distributiva que la última encuesta CASEN situó sobre 13 veces la diferencia de ingresos entre ricos y pobres $^{[5]}$.

En tal sentido, la observación del malestar en la sociedad no es sino otra forma de indicar la defraudación de expectativas, las que son finalmente remitidas a la conducción y administración política del Estado. Los condicionamientos estructurales de la sociedad contemporánea, limitan de modo efectivo los márgenes de intervención de la administración gubernamental y, en consecuencia, disminuyen las posibilidades de éxito en la disposición y distribución de condiciones de inclusión más efectivas. Las políticas públicas, concebidas como el principal mecanismo conducente a producir desde la esfera política, orientaciones de cohesión social ${ }^{[6]}$, están acotadas por una serie de limitaciones e indicaciones de los subsistemas de la sociedad.Paradojalmente, seindica quela aceptación de tales constreñimientos es una condición de su efectividad y eficiencia ${ }^{[7]}$, al mismo tiempo que un factor que incrementa la frustración en torno a las expectativas socialmente producidas $^{[8]}$.

[5] Mideplan. "Resultados Distribución del Ingreso e impacto distributivo del gasto social". Encuesta CASEN, 2006. En www. mideplan.cl. Un análisis más exhaustivo basado en el ingreso monetario per cápita (indicador 20/20), sitúa la brecha distributiva entre el $20 \%$ de hogares con menores ingresos y el 20\% de hogares con mayores ingresos en 16,51 veces. Ayala, Lorena. Pobreza y redistribución del ingreso en Chile. Universidad de Santiago de Chile, julio de 2006.

[6] Por cierto, esto no está garantizado, y todavía es posible sostener que las políticas públicas pueden contribuir, pero también no contribuir en la generación de cohesión social. Sobre esta discusión, en particular: Palma, Andrés. "Políticas públicas que contribuyen a la cohesión social". En Diálogos de políticas públicas. Revista Digital, FLACSO, Chile, N 1, Año 1, enero 2007. También, Palma, Andrés. "Las políticas públicas que no contribuyen a la cohesión social". Fundación internacional y para Iberoamérica de Administración y Políticas Públicas (FIIAPP). Santiago de Chile, 2008.

[7] Lahera, Eugenio. Introducción a las políticas públicas. Fondo de Cultura Económica, Santiago de Chile, 2008.

[8] Por cierto, también está la indicación contraria que sostiene que la cohesión social ha sido la dimensión olvidada de las políticas públicas. Peña, C. y Tironi, E. “La Miseria del Economicismo. Política, políticas públicas y cohesión social”. En Tironi, E. (Ed.) Redes, Estado y mercados. Soportes de la cohesión social latinoamericana. Uqbar, Editores, PNUD, Cieplan. Santiago de Chile, 2008: 23. 
Es en el ámbito de las políticas públicas orientadas a producir condiciones crecientes de inclusión social, donde se advierten incrementos en los niveles de incompatibilidad. Las actuales especificaciones técnicas y políticas imponen sobrecargas estructurales y operativas, tanto en el plano de su diseño, como en el de su implementación y resultados esperados. Desde esta perspectiva, las sobrecargas que soportan las políticas públicas, bajo la forma de externalidades esperadas, se pueden apreciar de modo particular en el plano de la racionalidad sistémica que guía su diseño y puesta en marcha. Es ampliamente aceptada, la observación que indica el carácter gravitacional que ha venido ejerciendo el sistema económico en el proceso de modernización de las últimas décadas ${ }^{[9]}$. Y resulta necesario distinguir que los "modelos desarrollo" no son otra cosa que programas ${ }^{[10]}$ orientados a producir la suficiente compatibilidad y concurrencia entre los sistemas de funciones, pero no es allí donde se encuentran los condicionamientos estructurales de la sociedad. Estos están determinados por el carácter autorreferencial de los sistemas. Los ciclos económicos no responden a la desigualdad distributiva -producen oscilaciones ${ }^{[11]}$, sino que a umbrales de acumulación y a las condiciones estructurales para sostenerlos ${ }^{[12]}$.

Por ello, el presente artículo ensaya una observación en torno a las condiciones estructurales sobre las que operan las relaciones sistema-asistema. Es en este plano, donde se especifican los condicionamientos que regulan la orientación de la sociedad $\mathrm{y}$, en particular, las dinámicas de acoplamiento entre los sistemas. Para ello, los sistemas preparan condiciones internas que orientan la toma de decisiones. En este caso particular, el ángulo de observación que se selecciona es el tipo de respuestas que ha

[9] Faúndez, Alejandra. “¿Nuevo enfoque de Políticas Sociales en Chile?” En Diálogos de políticas Públicas. Revista Digital, FLACSO, Chile, Nº1, Año 1, enero 2007.

[10] En el sentido de estrategias que seleccionan condiciones que se deben suponer o los resultados que se pueden esperar alcanzar, pero que pueden ser modificadas. Al respecto. Luhmann, Niklas. Sistemas Sociales. Lineamientos para una teoría general. Editorial Anthropos, Universidad Iberoamericana. Barcelona, 1998: 194; npp., 290.

[11] Al respecto, Londoño, Juan Luis y Székely, M. "Sorpresas distributivas después de una década de reformas: América Latina en los noventa". En Desarrollo más allá de la economía. Progreso económico y social en América Latina. BID, Washington, 2000.

[12] Thurow, Lester C. El futuro del capitalismo. Ediciones Ariel. Barcelona, 1996: 258 y ss. 
venido elaborando y ensayando el Estado de Chile en el marco del proceso modernizador vigente. Y el foco de análisis es el entramado decisional que soporta la orientación y racionalidad ${ }^{[13]}$ del diseño de políticas. Es decir, por una parte, el conjunto de premisas y criterios, articulados en semánticas particularizadas, que especifican y condicionan el tipo de decisiones que actualiza la administración política del Estado; y por otro lado, se calibra el papel que tiene el plano operativo de la toma de decisiones, y sus efectos en la orientación de las propuestas de intervención que implementa la gestión gubernamental.

Las preocupaciones teóricas que aquí se desarrollan, intentan avanzar en dos planos complementarios. Por cierto, el más evidente, es observar la dinámica de cambios que ha venido experimentando el Estado, porque con ello se evita excluir el problema de los efectos de estos procesos en la orientación y transformación de la sociedad. Y, por otro lado, se intenta disponer el complejo aparataje conceptual de la teoría de sistemas en el tamiz de los problemas aplicados. Las reflexiones conceptuales que aquí se desarrollan, tienen un carácter preliminar y su orientación es avanzar en la elaboración de un enfoque teórico y aplicado para la observación de las prestaciones que el Estado contemporáneo materializa a través de sus políticas públicas.

\section{Introducción: Estado y políticas públicas}

La sociedad inscribe las huellas de su temporalización mediante los conceptos que selecciona. Nada de trivial hay en los resultados de estos procesos. Los conceptos que la sociedad privilegia, no solo aglutinan las experiencias históricas, también buscan prescribir cursos posibles de acción ${ }^{[14]}$. Como un gran escultor, el tiempo de la sociedad desgasta, deshace y

[13] Cadenas, Hugo. "Un modelo de análisis para las políticas públicas". Revista de Estudios Interdisciplinarios, ASOSYLFF. Santiago de Chile, 2006.

[14] Koselleck, Reinhart. Futuro pasado. Para una semántica de los tiempos históricos. Ediciones Paidós. Barcelona, 1993. “La lucha semántica por definir posiciones políticas o sociales y en virtud de esas definiciones mantener el orden o imponerlo corresponde, desde luego, a todas las épocas [...]... los conceptos ya no sirven solamente para concebir los hechos de tal o cual manera, sino que se proyectan hacia el futuro" (pp: 111). 
precipita pérdidas conceptuales ${ }^{[15]}$. Hoy día resulta difícil hablar de igualdad o justicia social. Del mismo modo, la emergencia y eventual consolidación de las propuestas semánticas marcan el rumbo de las decisiones ${ }^{[16]}$. En consecuencia, restringen los márgenes de la comunicación, definen los temas y los problemas que se deben atender, pero también el tipo de respuestas y soluciones que se deben implementar. Es decir, efectivamente, acotan el horizonte de lo posible.

En el ámbito de la administración del Estado y los enfoques predominantes en materia de políticas públicas, no solo es evidente la transformación del lenguaje político-técnico, sino también la convergencia semántica y terminológica que lo acompaña. Recientemente se ha sostenido que los temas, conceptos y enfoques que caracterizan a las políticas públicas, se han terminado convirtiendo en una lengua franca, que no solo compromete a la lengua oficial, sino también al lenguaje corriente en la materia ${ }^{[17]}$. La aproximación al análisis de las políticas públicas es también una discusión en torno a la transformación del Estado ${ }^{[18]} \mathrm{y}$, con ello, de la sociedad ${ }^{[19]}$. Las orientaciones de estos procesos responden a los paradigmas políticotécnicos que dominan la agenda pública y regulan la toma de decisiones, y se corresponden con el conjunto de premisas y creencia que los agentes políticos y técnicos comparten en el ámbito del entorno público-estatal que diseña, acuerda, regula y gestiona las políticas públicas ${ }^{[20]}$. El interés por los modelos o paradigmas que guían la toma de decisiones en el aparato público no es nada nuevo ${ }^{[21]}$. Todavía constituye una referencia necesaria a la hora

[15] Yourcenar, Marguerite. El Tiempo, gran escultor. Alfaguara. Madrid, 2002.

[16] "Prejuzgan su propio futuro". Luhmann, Niklas. En: El amor como pasión. Ediciones Península. Barcelona, 1985.

[17] Lahera, E., 2008: 16.

[18] Santibáñez, Dimas y Zilveti, Maya. "Perspectiva de juventud e institucionalidad pública: condicionamientos contextuales." Revista Observatorio de Juventud. Perspectivas de Juventud y políticas públicas. Año 5, N 18. Julio, 2008.

[19] Jobert, Bruno. Estado, Sociedad, Políticas Públicas. Cátedra Unesco de Políticas Públicas, INAP, Universidad de Chile, LOM, 2004.

[20] Santibáñez, Dimas. "Modernización de la Gestión Pública: el Paradigma de la Racionalidad Económica y la semántica de la Eficiencia." Revista MAD (Revista Electrónica del Magíster Antropología y Desarrollo), N³. Septiembre, 2000.

[21] Jobert, B., 2004: 26. 
de abordar el análisis de las políticas públicas ${ }^{[22]}$. Paradigmas y modelos conforman mecanismos específicos que hacen posible los acoplamientos estructurales de los sistemas de funciones. Tienen una función restrictiva y facilitadora de las influencias recíprocas en las relaciones sistema-a-sistema ${ }^{[23]}$. Estas posibilidades aparecen en el margen interno del Estado, como autodescripciones -Estado neosocial, Estado regulador, Estado subsidiario- ${ }^{[24]}$, que incorporan referencias externas y preparan supuestos y premisas -bajo la forma de textos, teorías y modelos-, para operaciones posteriores ${ }^{[25]}$.

Por lo tanto, las convergencias semánticas y programáticas susceptibles de observar en tales recursos, indican un determinado tipo de integración inter-sistémica o, si se quiere, un ajuste histórico particular ${ }^{[26]}$, cuyo efecto se aprecia en el tipo de condicionamientos que se ejercen sobre la modulación ${ }^{[27]}$ de las políticas, la secuencialización de las decisiones y, en definitiva, sobre la orientación de las transformaciones sociales -lo cual, por cierto, incluye la transformación del Estado. A mi juicio, el espacio comunicativo que se estructura en torno a la formulación e implementación de políticas, constituye todavía en el presente, una clara expresión del inacabado y siempre problemático proceso de diferenciación de la sociedad. Puede indicar, por otro lado, la forma de la desdiferenciación operativa, si por ello se entiende el desencadenamiento de tensiones, contradicciones y conflictos, como efecto de las condiciones de auto-irritabilidad de los sistemas -bajo la forma de sorpresas y perturbaciones, o déficit de sincronización y coordinación-. Nada

[22] Por ejemplo, Madariaga, Hugo. “Modelos de Desarrollo Social y las políticas para la infancia." En: Infancia y Políticas Sociales. Análisis de los modelos de Desarrollo Social y de las Políticas de Protección de los Derechos del Niño. UNICEF-Universidad de Chile, 2002: 9-53.

[23] Luhmann, Niklas. El Derecho de la Sociedad. Ediciones Herder, Universidad Iberoamericana, México, 2005: 507 y ss.

[24] Por ejemplo, Faúndez, A., 2007: 17.

[25] Luhmann, N., 2005: 567 y ss.

[26] Luhmann, Niklas., La sociedad de la sociedad. Ediciones Herder, Universidad Iberoamericana, México, 2007: 478. Si por integración se entiende la reducción de los grados de libertad de los sistemas parciales.

[27] En el sentido de un cambio de tono dentro de la autorreferencia de criterios que orientan la toma de decisiones. Hofstadter, Douglas. Gödel, Escher, Bach. Un eterno y grácil bucle. Tusquets Editores. Barcelona, 1987. 
de esto podría tener importancia. Sin embargo, la adquiere cuando se busca producir conducción, coordinación y control social a través de la actuación pública estatal con el objeto de superar los déficits sociales que se observan, evitar las externalidades negativas que producen los sistemas de funciones o garantizar mayores condiciones de inclusión social.

El enfoque que el párrafo anterior insinúa, no desprende el análisis de las políticas públicas del problema de la transformación del Estado. No lo puede hacer porque las políticas públicas constituyen uno de los ámbitos privilegiados en el que operan las prestaciones de la política, cuando otros sistemas parciales requieren de decisiones vinculantes ${ }^{[28]}$. Y debido a ello, deben ser entendidas, también, como intervenciones destinadas a producir efectos de orientación, coordinación y cambio social. Desde mi punto de vista esto es todavía correcto, incluso cuando no es posible afirmar que la política y el accionar del Estado determinan o conducen los destinos de la sociedad ${ }^{[29]}$. En tal sentido, la racionalidad de las políticas públicas se estructura en función de la diferencia entre heterorreferencia y autorreferencia, en la medida que expresa el tipo de observación que la administración elabora para abordar la complejidad de la sociedad. En consecuencia, están orientadas a producir efectos de co-evolución, a partir de las particulares lecturas -procesamientos de información- que los equipos técnicos y políticos del Estado establecen respecto de las dinámicas y problemas de la sociedad. Es en este sentido que las políticas públicas deben ser observadas como opciones -en el sentido de equivalentes funcionales- que responden a condicionamientos estructurales seleccionados-y privilegiados-, en el marco de los procesos de transformación de la sociedad.

Como indiqué en la presentación de este texto, la particular manera que las burocracias profesionales y técnicas tienen para observar y procesar los déficits y desafíos que debe abordar la acción gubernamental responde, finalmente, a los paradigmas o modelos político-técnicos que comparten. No solo eso, ese conjunto de premisas y criterios, delimitan el abanico de opciones posibles para diseñar las respuestas o soluciones que las políticas

[28] Luhmann, Niklas. Teoría politica en el Estado de Bienestar. Editorial Alianza Univerdidad, Madrid, 1997: 95.

[29] Wilke, Helmut. "Capítulo 4: Formas de Autorientación de la Sociedad". En Teoría de Sistemas de las Sociedades Desarrolladas. Dinámica y Riesgo de la Autoorganización Social Moderna. Mimeo, 1993. 
públicas deben privilegiar. En consecuencia, los paradigmas político-técnicos, y las comunidades profesionales que los sostienen, operan restringiendo el ámbito de la comunicación y la deliberación ${ }^{[30]}$. La función de los contenidos paradigmáticos o discursivos ${ }^{[31]}$-semánticas y programas- es incrementar la probabilidad del éxito de los procesos de deliberación y negociación que involucra la toma de decisiones en el diseño de las intervenciones estatales. El éxito, sin embargo, no está asegurado como se puede atestiguar, por ejemplo, a propósito de la orientación que deben tomar las políticas de protección hacia los trabajadores o de la oportunidad de una administradora de fondos de pensiones de carácter estatal. De este modo, no es antojadizo indicar que en la medida en que se incrementa el proceso deliberativo, y en cierto sentido se dilata la indefinición, contamos con un indicador del grado de condicionamiento contextual de la sociedad y con ello de diferenciación. Simplemente, ya no resulta trivial imponer criterios.

Como ya se sabe, estos procesos intermedios de deliberación y negociación tienen un carácter crítico y estratégico en el plano de las dinámicas internas de las administraciones gubernamentales. Como ha apuntado Wilke, (1993) es en este plano en el que se encuentran los imperativos funcionales de los sistemas sociales parciales. Es en este plano, en el que se producen las condiciones adecuadas para los acoplamientos operativos. Y, desde una presunción teórica de tipo cooperativa, se estructura la expectativa de un incremento en los efectos de coordinación y co-orientación en el marco de las decisiones de intervención de la sociedad. Este tipo de dinámicas se desarrolla, de modo especial, en el entorno interno del Estado e involucra actores políticos, profesionales y técnicos, representantes de las más diversas reparticiones del entramado institucional del Estado, que operan incorporando las comunicaciones específicas de los entornos sociales que conectan. La complejidad de la sociedad, está reintegrada estructuralmente en la configuración institucional de la burocracia estatal.

[30] Sobre la distinción y relación recíproca entre paradigma y programa de acción ver Dammame, D. y Jobert, B., (1999). Para los autores los programas de acción constituyen las herramientas de diagnóstico y fórmulas de acción concretas, pero que deben estar constituidas en comunidades epistemológicas. "Estas comunidades epistemológicas constituyen una zona fronteriza por excelencia, donde se encuentran científicos, expertos provenientes del mundo de la decisión, los aportes de unos y otros, son indispensables para construir instrumentos pertinentes de política" (Ibid.: 5).

[31] Wilke, H., 1993. 
Desde mi perspectiva, sin embargo, los procesos de análisis y tratamiento de las opciones de políticas, ponen en evidencia no solo los condicionamientos estructurales en los que se desarrollan estas dinámicas, sino también sus tensiones, contradicciones y conflictos, al mismo tiempo que los mecanismos mediante los cuales se resuelven tales indefiniciones. Esto es así, porque las transformaciones que ha experimentado el Estado durante las últimas tres décadas, han terminado por configurar una contextualidad interna que integra cada vez mayores presupuestos estructurales, condiciones de incompatibilidad ${ }^{[32]}$ y sobrecargas de expectativas a la formulación de políticas públicas. ${ }^{[33]}$ Esta complejidad busca ser resuelta a través de la producción de programas decisionales que determinen la dirección, los límites y alcances de los esfuerzos públicos estatales, pues ya no resulta suficiente garantía de coherencia la coalición, el partido o la ideología política. Pero en ello, no deja de ocupar su sitio el peso relativo de los actores involucrados, así como de los argumentos técnicos y políticos que se esgrimen para ordenar secuencial y jerárquicamente la variedad de opciones producidas de modo contingente. Un incremento de la variedad de opciones, sistémicamente referidas, estimula dinámicas de aprendizaje reflexivo y la incorporación de criterios novedosos para la toma de decisiones. ${ }^{[34]}$

En consecuencia, sostengo que las nuevas complejidades que debe abordar el Estado contemporáneo, no provienen únicamente de los problemas que la sociedad indica como entorno del sistema político, sino de manera creciente desde su propio entorno interno. Esto es resultado de los cambios, reformas y modernizaciones que el Estado chileno ha venido experimentado en las últimas décadas y constituye la contextualidad en la que se formulan las políticas públicas. En lo que sigue, se desarrollan algunas consideraciones

[32] Santibáñez, Dimas. "Condicionamientos estructurales para la inclusión social: racionalidad económica y transformación del Estado. Requerimientos funcionales y paradojas". En: Nuevas Exclusiones en la complejidad social contemporánea. Ediciones MAD, Facultad de Ciencias Sociales Universidad de Chile, 2006. También, Jobert, B., 2004.

[33] Es decir, que toma la forma de una contradicción. Luhmann, N., 1998: 324 y ss.

[34] Wilke, H., 1993: 18. La variedad es una condición de la autoproducción de los sistemas. “Cuanto más grande la variedad de un sistema, tanto más difícil es deducir de una operación a otra, a partir del conocimiento de solo algunas de sus operaciones; tanto más sorpresas se producen que deben ser procesadas; tanto más largas las cadenas; tanto más tiempo necesita el sistema" (Luhmann, N., 2005: 422). 
conceptuales respecto del tipo de contextualidad interna que caracteriza el Estado contemporáneo y las consecuencias que ello genera en el plano de la toma de decisiones vinculadas al diseño de las políticas públicas.

\section{Contextualidad y condicionamientos estructurales}

\section{del Estado contemporáneo}

El objetivo de las breves líneas que siguen a continuación, es proponer un conjunto de indicaciones de tipo conceptual que refuercen los planteamientos desarrollados hasta aquí. Por cierto, se hacen en referencia a los principales ejes de cambio que ha venido experimentando el Estado chileno. La abstracción teórica encuentra su desenlace en el análisis aplicado.

Todavía es posible sostener que la imagen objetivo que orienta la toma de decisiones en materia de modernización y reforma del Estado, incluye criterios de contención del tamaño del aparato público, especialización en sus funciones y competencias, mejoramiento de la eficiencia en materia de gestión y una creciente profesionalización y tecnificación de su quehacer. Al mismo tiempo, se espera que la gestión pública mantenga una lógica económica estricta, sea capaz de diversificar su oferta y garantizar espacios cada vez más amplios para que el mercado actúe de modo autónomo bajo necesarios mecanismos de regulación. Sus desafíos, son apoyar el proceso de desarrollo promoviendo la competitividad y mejorando las condiciones de equidad social. ${ }^{[35]}$

Tales descripciones mantienen plena vigencia y son coherentes con las premisas paradigmáticas que han guiado la toma de decisiones, no solo en materia de reforma y modernización del aparato público, sino también en lo que dice relación con la puesta en práctica de las políticas públicas de carácter social. En función de los desafíos que supone sostener las prestaciones del Estado, las administraciones gubernamentales han venido implementando un conjunto de iniciativas que han involucrado: la diferenciación institucional y administrativa del aparato estatal -creación de institucionalidad-; el reforzamiento de las capacidades de prestación de la acción pública-estatal

[35] Boeninger, Edgardo. Políticas públicas en democracia. Institucionalidad y experiencia chilena 1990-2006. Colección Cieplan, Uqbar Editores. Santiago de Chile, 2007. También, French-Davis, Ricardo. Entre el neoliberalismo y el crecimiento con equidad. Tres décadas de política económica en Chile. Dolmen Ediciones. Santiago de Chile, 2001. 
-estrategias e instrumentos de intervención-; la modernización de la gestión pública; y la profundización de la democracia ${ }^{[36]}$. Finalmente, en el marco de la última década, los esfuerzos orientados a ampliar e incrementar el ámbito y la profundidad de la inclusividad, mediante la progresiva consolidación de un Sistema de Protección Social en el país, ha supuesto la implementación de una serie de iniciativas que incluye los ámbitos antes señalados. En este marco, por ejemplo, no es menor el esfuerzo que se viene realizando en favor de los niños y niñas del país a través del Programa Chile Crece Contigo o las expectativas que se empiezan a cifrar en torno a la reforma de la educación como estrategia de equiparación de oportunidades.

He sostenido en otras ocasiones, que el programa decisional que guía este tipo de modernizaciones y reformas, se estructura en función de dar continuidad y fortaleza al accionar del Estado en tres ámbitos de competencias o mandatos críticos: estabilización, promoción y compensación -y hoy progresivamente habría que añadir regulación-[37]. De este modo, por ejemplo, la creación o fortalecimiento de instituciones con estatuto de autonomía para efectuar acciones de regulación, modifica la diferenciación institucional de la burocracia estatal. La incorporación de agentes del mercado en la provisión de servicios o en la concurrencia para mejorar la competitividad estructural -concesiones en infraestructura-, introduce nuevos criterios de racionalidad en la toma de decisiones, pues no se puede desincentivar la participación estratégica de los actores económicos. Del mismo modo, la progresiva adopción de la perspectiva de derechos supone, entre otros aspectos, la flexibilización de los dogmas que han dominado la agenda gubernamental, la inclusión de racionalidades alternativas en materia de decisiones de política social y el arreglo de nuevas condiciones de coordinación en el entorno interno del Estado ${ }^{[38]}$.

[36] Santibáñez, D. y Zilveti, M., 2008: 44-45. También, Tomassini, L. y Armijo, M. Reforma y modernización del Estado. Experiencia y desafio. Instituto de Asuntos Públicos, Universidad de Chile, LOM. Santiago, 2002.

[37] Santibáñez, D., 2006. Los ámbitos de competencias pueden ser especificadas desde el plano del sistema total -funciones-, o desde un plano de las relaciones sistema-asistema -prestaciones-.

[38] Para una discusión en torno a los presupuestos de la perspectiva de derechos y su impacto en las políticas públicas, Erazo, Ximena, Martin, Ma Pía y Oyarce, H. (Editores). Políticas Públicas para un Estado Social de Derechos. Vol. I. Fundación Henry Dunant América Latina, LOM Ediciones. Santiago de Chile, 2007. 
Desde un punto de vista conceptual, el efecto neto de este tipo de transformaciones es la conformación de una contextualidad interna que condiciona la acción del Estado contemporáneo y, en particular, el accionar de sus equipos técnicos y políticos ${ }^{[39]}$. En síntesis, estos efectos se pueden resumir en las siguientes notas:

- Las respuestas que se diseñan e implementan, en el marco de una mirada de larga duración, tienen efectos de transformación estructural no programados que condicionan la complejidad interna del Estado y de la gestión pública, en orden a producir entornos internos diferenciados que refuerzan racionalidades particularizadas, aumentando con ello el déficit de coordinación y minimizando las probabilidades de coevolución ${ }^{[40]}$.

- En virtud de lo anterior, la gestión de las políticas públicas se ve autoenfrentada a una carencia de unidad de sentido que se expresa, por ejemplo, como asimetría entre criterios de tipo técnico y de tipo político, como restricciones para la coordinación, como desacople entre criterios de tipo económico y criterios de tipo sectorial y, sobre todo, como una sobrecarga en materia de expectativas.

- Frente a ello, la gestión gubernamental busca coordinar y orientar las actuaciones institucionales a través de definiciones políticas y programáticas, definiciones técnicas que especifican las características que deben alcanzar las políticas públicas en su diseño, instrumentos de gestión que indican el tipo de selecciones que se deben priorizar y los alcances que se pueden tener y, por cierto, instancias de análisis, deliberación y decisión de carácter formal e informal.

En consecuencia, y de acuerdo a lo adelantado en las primeras páginas de este trabajo, dos son los mecanismos que se deben considerar como componentes claves que hacen posible la toma de decisiones en torno a la formulación de políticas públicas. Por una parte, los procesos de deliberación y negociación que sostienen los equipos técnicos de las diversas reparticiones comprometidas en el diseño de políticas -integración de variedad de opciones-. Y, en segundo lugar, los criterios paradigmáticos y programáticos

[39] Santibáñez, D. y Zilveti, M., 2008.

[40] Wilke, H., 1993. También, Jobert, B., 2004. 
que guían el proceso de toma de decisiones -regulación y jerarquización de la toma de decisiones-. Por cierto, todo ello no asegura una total racionalidad, coherencia y efectividad, pero si garantiza condiciones mínimas de operación. Sus resultados específicos se pueden observar, de modo particular, en las referencias semánticas y programáticas que recurrentemente se actualizan para el diseño de políticas públicas y, de modo particular, en los objetivos, resultados esperados y características específicas que alcanzan las propuestas de implementación. El punto de vista que he venido sosteniendo aquí, es que en la medida que aumentan los presupuestos que guían estos procesos, la aparición de incompatibilidades y desajustes aumentan debido a la presión que ejercen formulaciones divergentes. El proceso deliberativo y formulador de políticas se hace más complejo lo cual, afortunadamente, comienza a ser evidente en el caso de las políticas orientadas a revertir las externalidades sociales negativas.

Es posible sostener, entonces, que un incremento en las dificultades para producir acuerdo en torno a las políticas públicas, supone la observación de incompatibilidades que obligan a análisis posteriores ${ }^{[41]}$. La incompatibilidad se observa en dinámicas de indefinición en materia de decisiones e introduce inseguridad respecto de los efectos. La observación de las incompatibilidades constituye un logro evolutivo, pues supone la condición de establecer la diferencia entre autorreferencia y heterorreferencia. Es decir, incrementar las condiciones internas para observar los efectos de los procesos en curso y preparar condiciones de respuestas antes no previstas o actualizadas, por ejemplo bajo la forma de avances adaptativos. Desde mi perspectiva, ello es posible, entre otras cosas, debido a que en el caso de la complejidad del Estado contemporáneo: la sociedad está reintegrada estructuralmente en la configuración institucional de la burocracia estatal. Ello hace posible la autoobservación y la indicación de los límites de los modelos vigentes para producir efectos políticamente legítimos -p.e. reducción de la pobreza y redistribución de ingresos ${ }^{[42]}$. En el presente, ello se ha venido resolviendo a través del diseño e implementación del sistema de protección social. Pero aquí todavía hay alternativas de enfoque y opciones de decisión y también

[41] Medido, por ejemplo, en términos de dilatación en la toma de decisiones -norma en la televisión digital-, dinamización de la comunicación -AFP estatal-, indefinición de montos financieros involucrados -sueldo ético, pensión solidaria-.

[42] Martner, G., 2007: 62 y ss. 
cuestionamientos respecto de las condiciones de gestión para producir los efectos esperados -ifallas de coordinación horizontal y vertical!_[43].

Las notas desarrolladas hasta aquí, explican la aparición de este tipo de comunicaciones. La formulación propuesta en este trabajo es que el Estado auto-produce su propia complejidad como efecto de la evolución de la sociedad. Es cierto que en el planteamiento sistémico, el Estado es especificado como la auto-descripción del sistema político y diferenciado de la administración pública y el gobierno. Sin embargo, en el marco de este planteamiento, por Estado comprendo el entramado institucional y organizativo sobre el que se sostiene la división del poder en el sistema político. Luhmann la especifica como Administración ${ }^{[44]}$ y el gobierno solo está en condiciones de conducir un ámbito cada vez más restringido de competencias y mandatos. La evolución del entramado institucional del Estado supone la progresiva inclusión de la diferenciación de la sociedad en el entorno interno del sistema de la política y, en consecuencia, el arreglo de condiciones estructurales para las relaciones sistema-a-sistema. Como logro evolutivo, supone la preparación de condiciones de irritabilidad frente a las comunicaciones de la sociedad, al mismo tiempo que la incorporación de criterios de racionalidad particularizados, ajustados a requerimientos sistémicos específicos. Bajo estas condiciones, se estructuran posibilidades para el desarrollo de dinámicas de acoplamiento/desacoplamiento o si se quiere dependencia/independencia, en las relaciones sistema-a-sistema ${ }^{[45]}$. Los propios sistemas, a partir de las perspectivas específicas que actualizan, modulan las dinámicas de independencia/dependencia ${ }^{[46]}$.

[43] Según el énfasis, protección social en el ciclo de vida, para el desarrollo de capital humano, social y cultural, para el desarrollo de capacidades o como universalismo básico. Faúndez, A., 2007.

[44] Luhmann, N., 1997: 62.

[45] Luhmann, N., 2007: 475.

[46] Sobre este tipo de dinámicas se elaboran las condiciones estructurales de la necesaria incoherencia de la acción estatal. Por ejemplo, Jobert, B., 2004: 43. 
La teoría de la determinación concéntrica de la sociedad -y las dinámicas de colonización inter-sistémicas-[47], enseñó la alta probabilidad de conexiones contradictorias y precarias en las relaciones sistema-a-sistema, al mismo tiempo que la probabilidad de pretensiones de regulación mediante el bloqueo de opciones y autonomías parciales, con el objeto de propiciar mejores condiciones operativas para sistemas específicos ${ }^{[48]}$. Al parecer, este tipo de dinámicas es particularmente crítico y característico para el caso de sociedades en procesos de transformación -modernización-, y todavía más, cuando parte de los esfuerzos están orientados a alcanzar los niveles de logro definidos por los organismos internacionales. La modernización está cada vez más sujeta a los requerimientos de los sistemas de funciones y sus conexiones de carácter global. Por lo tanto, la sostenibilidad de estos procesos exige regular la conectividad mundial de los sistemas de funciones, y por otro lado, mantener operativo el conjunto de presupuestos sobre los que se conducen las transformaciones en el ámbito regional o local de la sociedad. Esto último implica, también, regular el incremento de las expectativas.

Como ya se indicó anteriormente, el proceso de modernización privilegiado por nuestra sociedad ha supuesto un ajuste histórico particular. La tesis que se ha sostenido en otros trabajos, es que el proceso de modernización hasta ahora conocido se ha auto-impulsado a partir de un acoplamiento estricto -firme- entre política y economía en el plano de sus elementos -decisiones vinculantes/recursos financieros. Política y economía se han presupuesto mutuamente, y su operacionalización selectiva se ha dejado sentir de modo paradigmático en el entorno interno de la política y el Estado. Ello, en gran medida, debido a que el sistema de la sociedad opera sobre la expectativa de un acoplamiento estructural entre política y economía ${ }^{[49]}$. En la conformación del presupuesto, quedan integrados algunos de los requerimientos y condicionamientos económicos, por ejemplo, bajo

[47] Mascareño, Aldo. “Teoría de Sistemas de América Latina. Conceptos fundamentales para la descripción de una diferenciación funcional concéntrica". Revista Persona y Sociedad.

[48] Tal vez sea oportuno indicar que los modelos de desarrollo operan sobre la base del supuesto de la coordinación inter-sistémica y la concurrencia de efectos y externalidades en función de propósitos previamente diseñados.

[49] Por ejemplo a través de impuestos y disposición de recursos. Luhmann, N., 2007: 618. 
la forma de reglas que especifican los umbrales de gasto. La evolución y complejización de la sociedad, supone la posibilidad del debilitamiento de los acoplamientos firmes que han estructurado los condicionamientos que han operado en los procesos de modernización. No cabe duda que en ello ha jugado un papel clave la producción de reflexión que ha indicado, por ejemplo, la carencia de política en la formulación de políticas ${ }^{[50]}$. Y en esa dirección se visualiza el esfuerzo por consolidar un sistema de protección social institucionalmente fundado -legalmente acoplado-.

Por cierto, los acontecimientos nos recuerdan la fragilidad de los soportes que sostienen las expectativas de la política por producir un orden legítimamente sustentado - p.e. mediante la fórmula de la cohesión social-. ${ }^{[51]}$ Todavía es posible observar el efecto condicionante que ejerce el acoplamiento entre política y economía, no solo como limitación estructural de la sociedad, sino también como orientador de los alcances de las políticas públicas para producir los efectos que se esperan. Como he indicado a lo largo del texto, la modulación de las políticas públicas responde a los paradigmas políticotécnicos que orientan la toma de decisiones y el peso específico de los equipos técnicos que participan en las instancias de diseño y coordinación. La consecuencia neta de estos procesos se observa en las características específicas que adquieren esas formulaciones $\mathrm{y}$, en consecuencia, en el tipo de selecciones que se actualizan y también en la observación de las opciones que quedan bloqueadas. Ello como resultado de la aceptación de un conjunto de condicionamientos estructurados en el contexto de un ajuste histórico particular.

Es probable, entonces, que el malestar, alojado en el seno de las paradojas, no sea otra cosa que la expresión incomunicable de la paralización de opciones que se observan como posibles y deseables. Aquellas que alimentan legítimas expectativas de inclusión en el conjunto de prestaciones que la sociedad produce.

[50] Peña, C. y Tironi, E., 2008: 25 y ss.

[51] Todavía lejos de alcanzar condiciones de igualdad compleja, las opciones se restringen a la igualdad de oportunidades. Ello, al parecer, porque las determinaciones estructurales que producen los sistemas de funciones - p.e. orientación a la acumulación para el caso de la economía monetaria-, minimizan las probabilidades de estructurar condiciones distributivas justas en sociedades regionales débiles en su diferenciación sistémico-institucional. En torno a esta discusión, Martner, G., 2007: 96 y ss. 


\section{Políticas públicas: orientaciones y racionalidad}

Lo dicho hasta aquí, postula el carácter siempre improbable de las respuestas que el Estado diseña en el plano de alcanzar el óptimo que la expectativa política y social comunica. Paradojalmente, ello es fruto de la evolución de la sociedad que incluye cada vez más presupuestos sobre los cuales desenvolver sus dinámicas. Los acoplamientos firmes producen condicionamientos específicos y estrictos en las relaciones sistema-a sistema. La particular incorporación que el Estado contemporáneo realiza de los efectos sistémicos, se procesa y resuelve mediante los mecanismos operativos que ha dispuesto para ello. Paradigmas y equipos técnicos que los sostienen configuran un entramado decisional que busca producir las orientaciones, compatibilidades y coordinaciones que se ajustan a los criterios que seleccionan como prioritarios. Los procesos de negociación involucrados en la formulación de políticas, especifican el peso relativo de los actores políticos y equipos técnicos involucrados, determinando el tipo de modulación que finalmente toman las propuestas públicas de las administraciones gubernamentales.

En el caso de nuestro país, y junto con el avance de la presente década, el diseño de políticas públicas ha conocido criterios alternativos o complementarios. La adopción del enfoque de derechos, en el ámbito de las políticas sociales, se ha indicado como un cambio paradigmático respecto de la lógica de la compensación. ${ }^{[52]}$ Sin embargo, todavía las reformas estructuradas en el marco de la protección social no comprometen el conjunto del aparato del Estado y, por el contrario, parte de sus esfuerzos se ven sometidos a enormes sobrecargas y exigencias. Desde mi perspectiva, esto se debe a que en el marco de los procesos de formulación de políticas se deben encontrar soluciones que satisfagan las expectativas que incorporan los equipos técnicos de las diferentes reparticiones involucradas en el diseño de las acciones gubernamentales. El peso relativo del visto bueno presupuestario constituye, sin duda, un orientador efectivo de las características que adquieren finalmente las propuestas de implementación de las políticas. La subordinación de criterios, se hace efectiva en el proceso intermedio de deliberación entre los equipos técnicos que determinan los recursos a intervenir y aquellos de tipo sectorial.

[52] La Protección Social de cara al futuro: Acceso, Financiamiento y Solidaridad. CEPAL Naciones Unidas. Montevideo 2006. 
En consecuencia, y quizás por ello, el diseño de políticas todavía no abandona la lógica de la compensación, puesto que tanto la equiparación de oportunidades como la protección frente a los riesgos de las externalidades, son semánticas que refuerzan una estructura decisional que opera bajo la forma de una subordinación negativa en el plano de las operaciones ${ }^{[53]}$-es decir, deja de hacer o hace lo justo, para no intervenir los umbrales que determina la dinámica económica-. La recurrente denuncia que comunica el déficit de coordinación, se equivoca cuando refiere este problema a un mejoramiento en el plano de las operaciones y los vínculos institucionales. La inestabilidad de la coordinación, constituye un condicionamiento estructural, ya que no se puede garantizar la sincronización de todas las operaciones que se despliegan con la puesta en marcha de las comunicaciones que quedan incluidas en las propuestas de políticas. Ello incluye la comunicación que indica la necesidad de una política para un ámbito determinado de problemas. Cada plano o contexto de la sociedad, reacciona a ritmos diferenciados frente a estos estímulos, en función de las condiciones estructurales que presentan para incluir o excluir las posibilidades que se abren ${ }^{[54]}$.

Las desiguales condiciones estructurales que presentan los sistemas diferenciados, suponetambiénla presencia-oausencia-dedispositivoscapaces de anticipar efectos y por lo tanto, reacciones, bajo la forma de expectativas. En función de esta condición es posible el desarrollo de mecanismos orientados a bloquear opciones alternativas. Desde mi perspectiva, este tipo de subordinación, que en el marco del análisis sistémico se indica como un ajuste de tipo dependiente, se puede observar en algunas de las definiciones paradigmáticas que guían o condicionan el diseño de políticas públicas. Los criterios allí acordados, especifican el tipo de modulación posible de las propuestas. Su coherencia suele prescribirse en función de los criterios que la lengua oficial ha definido como parámetros consensuados.

En consecuencia, resulta oportuno diferenciar dos planos de análisis que exponen las fórmulas de limitación. En primer lugar, el condicionamiento semántico que especifica el margen de posibilidad de las decisiones en materia de políticas orientadas a producir reversos en las externalidades

[53] Offe, Clauss. Contradicciones en el Estado de Bienestar. Editorial Alianza, Madrid, 1990. También, Santibáñez, D., 2006.

[54] "También las ondas de resonancia en los sistemas acoplados pueden ser de diversa longitud y complejidad", Luhmann, N., 2005: 511. 
sociales negativas. Y en segundo lugar, los criterios técnicos que se incluyen en el diseño de las políticas.

En función de lo anterior, parece pertinente utilizar como punto de partida la siguiente referencia: dado que la racionalidad técnica opera de modo paralelo a la racionalidad política, las políticas públicas de calidad ${ }^{[55]}$ deben responder a requerimientos paradigmáticos particulares. Al respecto, nos interesa destacar solo dos aspectos que ejemplifican lo indicado en el párrafo anterior:

- Las políticas públicas deben estar acotadas por márgenes financieros, pues deben dar estabilidad a los equilibrios macroeconómicos básicos: esto significa que deben permitir la necesaria consolidación del equilibrio fiscal, al mismo tiempo que no deben dañar los objetivos de competitividad y equidad.

- En segundo lugar, a través de las políticas públicas el accionar del Estado debe estar orientado a contribuir en la generación de externalidades y masas críticas que converjan con los procesos de modernización social y económica.

Ambas definiciones describen parte de lo que se ha venido indicando: indican el tipo de premisas que el paradigma tecno-político refuerza con el objeto de delimitar el margen de decisión y posibilitar un tipo de compatibilidad entre la acción del Estado y los sistemas en el entorno. En lo que sigue, se proponen algunas notas analíticas relativas a las consecuencias que se derivan de esta estructura decisional para el diseño de políticas. Tal como he insistido a lo largo del texto, el objetivo de ello es identificar el tipo de condicionamientos que operan y prescriben los cursos de acción gubernamental, al mismo tiempo que imprimen una particular orientación a las políticas públicas y a la implementación de sus programas. Es decir, privilegiando ciertas opciones y bloqueando otras.

La semántica de la Estabilidad. Política y economía encuentran en la semántica de la estabilidad, un punto de convergencia para la estructura de sus acoplamientos. No es parte de los propósitos indagar en torno a las huellas históricas de esta convergencia, pero si es posible afirmar que esta

[55] Lahera, E., 2008: 38. 
semántica constituye, a estas alturas, un condicionamiento de tipo estructural, pues especifica el tipo de expectativas que se han condensado a lo largo del tiempo en relación con la actuación del Estado ${ }^{[56]}$. No hay margen para desbordar las indicaciones que quedan contenidas mediante esta fórmula. En este contexto, la potencial solución de las externalidades sociales negativas, se enfrenta a una estructura teórica y discursiva que ordena jerárquicamente la toma de decisiones, condicionando, incluso, el tipo de comunicaciones -informaciones, acciones, "señales", etc.- que se pueden efectuar. Con ello, se establece el umbral máximo de lo posible o el umbral mínimo tolerable de riesgos. En el caso de nuestro país, esto se ha tematizado largamente de la siguiente manera: solo es posible generar una "igualdad en la medida de lo posible" y de tipo incremental ${ }^{[57]}$.

En consecuencia, desde el ángulo del problema del riesgo para la lógica económica, en "la medida de lo posible" significa que en el marco de una economía de mercado no es posible propiciar mayores niveles de igualdad en el corto plazo, pues se corre el riesgo de “....ahogar el principal motor del progreso económico y material", ya sea por déficits fiscales o cargas impositivas inhibidoras de la inversión ${ }^{[58]}$. Es decir, no se pueden sacrificar los equilibrios macroeconómicos como condiciones de crecimiento y competitividad -esto es un riesgo demasiado alto- ${ }^{[59]}$.

En consecuencia, se ha elaborado un discurso de segundo orden con una alta resonancia en los entornos políticos, sociales y culturales que busca actuar a modo de una estructura de contención de las demandas sociales por mayor igualdad, al mismo tiempo que busca propiciar efectos de empalme con los entornos relevantes y, en consecuencia, un alineamiento implícito con las premisas del paradigma hasta ahora vigente. Me refiero al discurso de la

[56] Y también respecto de las políticas públicas, pues ellas deben alcanzar grados importantes de estabilidad o resoluteness, o capacidad para mantenerse en el tiempo. Boeninger, E., 2007: 129 y ss.

[57] La continuidad y permanencia de este discurso paradigmático tiene ya larga data. Al respecto, Vial, J., 1998.

[58] Vial, J., 1998: 193-195.

[59] Martner, G., 1999. Dicho en los términos de Offe, C., (1990), “...en la subordinación negativa, el dominio del sistema económico, depende de que puedan estabilizarse las fronteras entre los respectivos sistemas, de manera que el sistema económico pueda evitar interferencias..." (Ibid.: 46). También, Lahera, E. (2008). 
equidad en las oportunidades ${ }^{[60]}$, el que desde una perspectiva técnica se ha descrito como: la "equidad no puede ser entendida sino como el ofrecimiento de oportunidades efectivas para que todos los integrantes de la sociedad puedan desarrollarse como ciudadanos y puedan participar de los frutos del progreso económico y social en base a su propio esfuerzo" ${ }^{161]}$.

Por lo tanto, lo que resulta relevante destacar es que sobre la base del entramado estabilidad/equidad, queda articulado un tipo de orientación de largo plazo para los procesos de transformación de la sociedad. Bajo la fórmula de la equidad de oportunidades es posible identificar dos tipos de premisas basales que lo sostienen. En primer lugar, la sociedad se tematiza como un abanico recursivo de oportunidades de progreso para los individuos. En estricto rigor, el principal mecanismo generador de posibilidades es el mercado, el cual, por lo demás, cuenta con dispositivos generadores de incentivos para el esfuerzo personal. Por lo tanto, en segundo lugar, el discurso de la equidad de oportunidades basa su efectividad en la interpelación a la motivación de logro de las personas, lo que en otros términos se puede tematizar como un traslado de los riesgos sistémicos a las decisiones personales ${ }^{[62]}$. Esto último, especifica los alcances que pueden tener los esfuerzos de intervención en materia de inclusión: generar condiciones mínimas, proveer incentivos y, por sobre todo, excluir, desincentivos y sus consiguientes efectos perversos.

Para los efectos de este trabajo no es posible profundizar en torno a los presupuestos que aquí quedan contenidos. La dinámica de la sociedad los desborda, y lo que hoy puede resultar una condición mínima para la inclusión social[63], mañana puede constituir un condicionamiento de la exclusión. La provisión de condiciones mínimas queda siempre al rezago de la complejidad de la sociedad y todo indica que producir el ajuste entre la

[60] Como ya se indicó, la equidad de oportunidades aparece como un equivalente frente a otras opciones: igualdad compleja o, en un punto intermedio, igualdad de trato. $\mathrm{Al}$ respecto, Martner, G., 2007.

[61] Al respecto, De Gregorio, J. y Landerretche, O., 1998: 153.

[62] Por cierto, el modelo de la protección social, busca en este sentido, proveer de estructuras sistémicas que protejan frente a los riesgos estructurales más evidentes y críticos: salud, empleo, primeros y últimos años de vida.

[63] En torno a este tipo de formulación, Mascareño, Aldo y Thezá, Marcel (2007). “Jóvenes y perspectiva de derechos: un acercamiento desde el marco de las condiciones mínimas". En "La juventud en perspectiva de derecho". Revista Observatorio de Juventud. Año 4, Número 13. Instituto Nacional de la Juventud. Santiago de Chile: marzo 2007. 
ampliación de las prestaciones de la sociedad y las expectativas que sobre ellas se estructuran, no solo requiere tiempo, sino también un efectivo incremento de las condiciones.

Dado que el problema anterior supera los propósitos de este texto, nos resulta suficiente, por el momento, reforzar las siguientes ideas que operan condicionando el diseño de políticas:

- Las definiciones técnicas que se estructuran en torno a la semántica de la estabilidad, indican los riesgos de conductas ampliamente inclusivas en materia de política pública, en tanto pueden terminar constituyendo una fuente de desequilibrios que impriman mayores grados de inestabilidad perjudicando las posibilidades de crecimiento $^{[64]}$.

- Por lo tanto, desde un punto de vista financiero, los esfuerzos estatales no pueden ser concurrentes en el tiempo si no están garantizados los mecanismos de financiación. De ahí que la semántica de la sociedad prefiera hablar de igualdad de oportunidades o de equidad.

- Elcriteriocompensatoriotodavíapresenteenlasemánticadelaprotección, se concentra en la creación de condiciones de seguridad mínimas. La expectativa formulada al futuro es que, para ciertos segmentos de la población, cada individuo desarrolle sus potencialidades y estrategias de inserción social, probablemente sobre la base de la expectativa de un mejoramiento convergente de las condiciones sociales que acompañan la motivación de logro.

- En consecuencia, el núcleo crítico del paradigma político-técnico no se encuentra en la restricción presupuestaria -sería solo un condicionamiento-, sino en la orientación y la racionalidad que deben adoptar pues, sobre ellas, los equipos técnicos estructuran altas expectativas en términos del conjunto de resultados que se esperan alcanzar. Solo así se puede entender la expectativa de producir efectos concurrentes en el contexto de la diferenciación de la sociedad.

[64] Vial, J., 1998; Marcel, M. y Tohá, C., 1998. 
Generación de externalidades. No resulta del todo transparente lo que se intenta indicar cuando se formula la expectativa de generación de externalidades por parte de las políticas. Sobre la base de trabajos aplicados ${ }^{[65]}$, recientemente desarrollados, es posible advertir aquí que en el plano de los resultados esperados $y$, en función de un criterio de eficiencia, el diseño de políticas adiciona una sobrecarga de efectos. El paradigma técnico-político tiende a optar por prestaciones que en su diseño integren un carácter multifinal, pues las acciones contempladas en ellas debieran tener la capacidad de generar externalidades positivas en ámbitos o sectores complementarios. Es decir, efectos concurrentes que estimulen cambios en el corto plazo o sostengan los procesos de transformación o modernización en el mediano y largo plazo.

Por cierto, ello en gran medida es resultado de la evidencia de la complejidad de la sociedad. Al parecer la complejidad de los déficits sociales o de las condiciones estructurales que sostienen las exclusiones, no se pueden resolver a través de medidas específicas o acciones aisladas ${ }^{[66]}$. Frente a ello, las políticas sociales han avanzado en la perspectiva de elaborar propuestas integrales orientadas a producir procesos de cambio sinérgicos. La perspectiva de la equifinalidad es distinta, sin embargo, a lo que aquí se ha descrito como políticas y programas de carácter multi-final. Esto último puede ser indicado como propuestas que incluyen acciones que deben producir efectos colaterales para propósitos distintos. Este es el caso del tipo de opción privilegiada en un ámbito de la educación parvularia: el aumento de la oferta de educación parvularia, a través de la construcciones de nuevas salas cunas y jardines infantiles, no está orientada únicamente por la racionalidad educativa, pues su externalidad esperada es favorecer el incremento de la participación laboral femenina.

Al parecer, las teorías o modelos en boga, tienden a maximizar los resultados de la intervención estatal provocando una creciente inclusión de intereses y expectativas ${ }^{[67]} y$, en consecuencia, sobrecargando las posibilidades de eficacia e impacto de las iniciativas gubernamentales. Una consecuencia específica de ello es que las orientaciones de sentido comunicadas, tienden a perder claridad y consistencia.

[65] Me refiero al Estudio Determinantes de la demanda por educación parvularia. Convenio BID, Mineduc, Programa Pulso, Facultad de Ciencias Sociales, Universidad de Chile.

[66] Lahera, E., 2008.

[67] Al respecto, Luhmann, N. 1997. 
La expansión del ámbito de prestaciones vinculadas a una política pública específica constituye, por defecto, una fuente de problemas, pues el éxito o efectividad de la prestación depende de si los medios del sistema emisor -en este caso, las reparticiones responsables de la implementación y gestión de las políticas públicas-, posibilita un acoplamiento efectivo en los contextos que se intervienen. Al respecto, no se debe perder de vista que en uno de sus planos las políticas públicas son, efectivamente, propuestas comunicativas, cuya comunicabilidad se garantiza mediante la disposición de recursos -fundamentalmente financieros-, al mismo tiempo que mediante la formulación de expectativas de cambio social y/o cultural. Demás está decir, entonces, que cuando la expectativa de la política pública es producir efectos -fines- múltiples, que responden a criterios de racionalidad distintos, por mucho que puedan entenderse como complementarios, las posibilidades de vinculación disminuyen, ya que el resultado neto del esfuerzo público-estatal es una complejidad debilitada desde el punto de vista de las poblaciones que responden a ella.

Este tipo de configuración, afecta de modo particular uno de los principios que orienta el diseño de políticas. Si por pertinencia, se entiende el grado de correspondencia que puede existir entre los requerimientos o necesidades de los grupos prioritarios y los mecanismos, procedimientos y recursos que se seleccionan para su satisfacción ${ }^{[68]}$, entonces la nocorrespondencia supondría una política pública debilitada en este plano, y supondría que en el campo de sus efectos, no solo generaría altos niveles de frustración en los equipos técnicos responsables de sus diseños, sino también consecuencias no esperadas como producto de las respuestas y decisiones que los ciudadanos actualizan a partir de ello. La pertinencia no puede sostenerse exclusivamente sobre la base del análisis de la necesidad y oportunidad de la política, sino que debe incluir una evaluación del grado de receptividad que tales propuestas generarán en la orientación de los resultados que se propone.

También es posible que genere un déficit de coherencia, si por coherencia se entiende algo más que la consistencia que presenta una política

[68] Al respecto, Caracterización y análisis de la política social dirigida a los jóvenes. Grupo de trabajo interministerial de Juventud, 1999. 
pública respecto del mandato ético-político que la sostiene ${ }^{[69]}$. La coherencia debe ser también una medida interna en el sentido del tipo de propuestas comunicativas que los programas formulan a sus entornos y con ello el tipo de expectativas que estimulan. Un déficit de coherencia puede incrementar las condiciones para efectos no esperados.

Por cierto, la complejidad de las políticas públicas no se reduce a los criterios aquí expuestos. Estos constituyen solo dos ejemplos del tipo de condicionamientos que quedan integrados como mecanismos que operan limitando el espectro de opciones y orientando el diseño de las propuestas de intervención. Resulta necesario seguir avanzando en el análisis de los presupuestos que quedan integrados en este tipo de formulaciones políticotécnicas. Esta tarea seguirá siendo gravitante no solo porque las políticas públicas están evidentemente incluidas en la dinámica de la sociedad -en tanto comunicaciones-, sino porque producen efectos de transformación de la sociedad que no siempre se pueden anticipar o que se orientan en direcciones no esperadas que refuerzan los malestares que la propia sociedad comunica. Ya se sabe que no siempre conducen a superar los déficits que la sociedad observa.

Frente a las restricciones y condicionamientos que la sociedad empieza a conocer como resultado de la autoobservación, la emergencia de variedad es una buena noticia. La conducción público-estatal se ve sometida a nuevas complejidades y debe orientar los diseños de políticas, considerando la contextualidad plural de la sociedad. En este sentido, para quienes consideramos necesario multiplicar los ángulos de observación y comunicación, nada mejor que recordar el imperativo ético formulado por un célebre constructivista: actúa siempre como para aumentar el número de alternativas $^{[70]}$.

[69] Ibid.

[70] Foerster, Heinz von. "Principios de autoorganización en un contexto socioadministrativo". En: Pakman, M. (Ed). Las semillas de la cibernética. Obras escogidas. Editorial Gedisa. Barcelona, 1996: 139. 